\title{
Limiting Performance Analisys of a Head Protection Helmet Using Multicriteria Control Optimization *
}

\author{
P.P. Moita ${ }^{\mathbf{a}, \mathbf{b}, \mathbf{d}}$, Miguel Antunes ${ }^{\mathbf{a}}$, J.F.A. Madeira ${ }^{\mathbf{b}, \mathbf{c}}$, J.E.B. Cardoso ${ }^{\mathbf{b}}$, A.J. Valido ${ }^{\mathbf{a}, \mathbf{b}, \mathbf{d}}$
}

\begin{abstract}
In this paper the limiting performance analysis of a head protection helmet is performed. A discrete model of the human head is used. A multicriteria optimum control problem is formulated in order to minimize the risk of injuries in case of impact. Several injury criteria are minimized and are required to remain below a safety threshold value. The optimal control force acting on the head is found. The optimal control force is determined by nonlinear programming. The equations of motion are integrated at-once, as it is typical for static response, instead of the traditional step-by-step integration.
\end{abstract}

Keywords- Optimal Control; Nonlinear Programming, Multicriteria; Head Injury

\section{INTRODUCTION}

According to the report provided by the World Health Organization [2], about 1.24 million people lost their lives as a result of road accidents, $23 \%$ of this number being motorcyclists. This type of accident is identified as the eighth cause death toll at the global level in 2010 [1].

Motorcyclists are considerably less protected in the event of an accident than users of some other vehicles, such as drivers and passengers of cars or trucks. This is due to the fact that, in the case of car users, they have a range of protective devices and equipment, such as a seat belt, air bag, neck support and the car's own structure, whereas a motorcyclist only has as a major means of protection the safety helmet.

Head injury following a road accident is one of the most frequent injuries in motorcyclists, occurring in about $66.7 \%$ of the cases, being the second most vulnerable area of the body to sustain the injury, only surpassed by the legs [3]. The protection of the head, therefore, is an aspect of extreme importance to guarantee some safety in case of accident, and the development and study of means of protection is essential for the improvement of such protection devices.

The limiting performance analysis of a head protection system can be obtained by finding the solution of an optimum control problem in which the control variable is the force acting on the driver's head, in order to minimize its risk of injury. Since the control force is generic, not representing any predetermined design, the limiting performance analysis measures the limits on the improvements of the protection system with respect to the prescribed performance criteria. The performance of such a system can be seen as a benchmark to which the performance of a real restraint system can be compared.

a CDP2T, ESTSetúbal, IPS,Campus do IPS, Estefanilha, 2914-761 Setúbal, Portugal

b IDMEC - Instituto Superior Técnico, Universidade de Lisboa, Av. Rovisco Pais, 1049-001 Lisboa, Portugal

c Departamento de Matemática, ISEL, IPL, Rua Conselheiro Emídio Navarro, 1949-014 Lisboa, Portugal

d CENTEC - Instituto Superior Técnico, Universidade Técnica de Lisboa, Av. Rovisco Pais, 1049-001 Lisboa, Portugal

\section{HUMAN HEAD RESPONSE ANALYSIS}

\section{A. Human Head Model}

Figure 1. Human Head Model

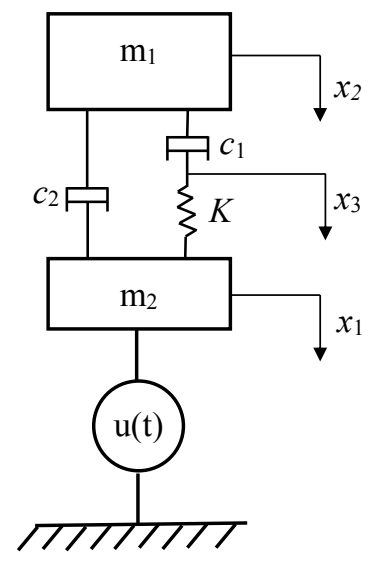

The model used to simulate the behavior of the human head in case of impact against a rigid surface, while the head is protected by a safety helmet, is the Translational Head Injury Model (THIM), as presented in reference [4].

In this model, the masses $\mathrm{m}_{1}=0.45 \mathrm{~kg}$ and $\mathrm{m}_{2}=4.09 \mathrm{~kg}$ represent the mass of the portion of the skull being impacted and the mass of the brain and the remaining bones of the skull, respectively, and where the sum of these masses provides the total mass of the head. These masses are connected to one another by a spring of stiffness $\mathrm{K}=13.5 \times 10^{6} \mathrm{~N} / \mathrm{m}$ and dampers with damping coefficients of, $\mathrm{C}_{1}=17 \times 10^{3} \mathrm{Nsm}^{-1}$ and $\mathrm{C}_{2}=157.6$ $\mathrm{Nsm}^{-1}$, which represent the mechanical properties of the skull bones and the dissipative properties of the brain, respectively. The element $\mathrm{u}(\mathrm{t})$ represents the action of the helmet on the head, more specifically, $u(t)$ is the control force generated by the inner lining, as it deforms in response to the impact.

\section{B. Response analysis}

The dynamic equilibrium equation of each mass can be arranged in matrix form as:

$$
\boldsymbol{M}^{t} \ddot{\boldsymbol{U}}+{ }^{t} \boldsymbol{C}_{S}{ }^{t} \dot{\boldsymbol{U}}+{ }^{t} \boldsymbol{K}_{S}{ }^{t} \boldsymbol{U}={ }^{t} \boldsymbol{u}
$$

where $\boldsymbol{M}$ is the mass matrix, ${ }^{t} \boldsymbol{C}_{S} \equiv{ }^{t} \boldsymbol{C}_{S}\left({ }^{t} \dot{\boldsymbol{U}}\right),{ }^{t} \boldsymbol{K}_{S} \equiv{ }^{t} \boldsymbol{K}_{S}\left({ }^{t} \boldsymbol{U}\right) \quad$ are, respectively, the damping and stiffness matrices, ${ }^{t} \boldsymbol{u}$ is the loading vector and ${ }^{t} \boldsymbol{U},{ }^{t} \dot{\boldsymbol{U}},{ }^{t} \ddot{\boldsymbol{U}}$ are respectively the displacement, velocity and acceleration vectors, all the quantities defined at time $t$. 
For temporal modeling, finite elements of dimension $\Delta t$ were considered, selecting hermitean cubic elements to model the displacements and quadratic lagrangean elements to model the loading, extending the algorithm given in [5] to the case of nonlinear systems. By taking the time derivative of the Equation (1) by one hand, and by another hand its integration once and then twice with average values of stiffness and damping in $\mathrm{t}$ given as

$$
\begin{aligned}
\overline{\boldsymbol{K}} & \equiv{ }^{t+\Delta t / 2} \boldsymbol{K}_{S}\left({ }^{t} \boldsymbol{U}+{ }^{t} \dot{\boldsymbol{U}} \Delta t / 2\right) \\
\overline{\boldsymbol{C}} & \equiv{ }^{t+\Delta t / 2} \boldsymbol{C}_{S}\left({ }^{t} \dot{\boldsymbol{U}}+{ }^{t} \ddot{\boldsymbol{U}} \Delta t / 2\right)
\end{aligned}
$$

one obtains four equations that combine to give the dynamic finite time-element equation as

$$
D_{S}^{e} z^{e}=\boldsymbol{u}^{e}
$$

where

$$
\begin{aligned}
& \boldsymbol{D}_{S}^{e}=\left[\begin{array}{ccc:ccc}
{ }^{t} \boldsymbol{K}_{S} & { }^{t} \boldsymbol{C}_{S} & \boldsymbol{M} & \mathbf{0}_{n \times n} & \mathbf{0}_{n x n} & \mathbf{0}_{n \times n} \\
\hdashline{ }^{t} \boldsymbol{D}_{S_{11}}{ }^{t} \boldsymbol{D}_{S_{12}} & \mathbf{0}_{n \times n} & { }^{t+\Delta t} \boldsymbol{D}_{S_{11}} & { }^{t+\Delta t} \boldsymbol{D}_{S_{12}} & \mathbf{0}_{n \times n} \\
{ }^{t} \boldsymbol{D}_{S_{21}}{ }^{t}{ }^{t} \boldsymbol{D}_{S_{22}} & \mathbf{0}_{n \times n} & { }^{t+\Delta t}{ }^{\boldsymbol{D}_{S_{21}}}{ }^{t+\Delta t}{ }^{+} \boldsymbol{D}_{S_{22}} & \mathbf{0}_{n \times n}
\end{array}\right] \\
& u^{e}=\left\{\begin{array}{r}
{ }^{t} \boldsymbol{P} \\
t+\Delta t / 2 \\
{ }^{t+\Delta t} \\
{ }^{P}
\end{array}\right\}
\end{aligned}
$$

and

$$
\begin{aligned}
& \boldsymbol{z}^{e}=\left({ }^{t} \boldsymbol{z},{ }^{t+\Delta t} \boldsymbol{z}\right) \\
& { }^{t} \boldsymbol{z}=\left({ }^{t} \boldsymbol{U},{ }^{t} \dot{\boldsymbol{U}},{ }^{t} \ddot{\boldsymbol{U}}\right)
\end{aligned}
$$

In Eq. (4) $n$ stands for number of space degrees-of-freedom and ${ }^{t} \boldsymbol{D}_{S_{j k}}$ are functions of $\overline{\boldsymbol{K}}, \overline{\boldsymbol{C}}, \boldsymbol{M}$.

Equation (3) may be solved step-by-step, i.e., element-byelement in time, or assembled to be solved at-once [9]. In this case, we have to assemble for a total time interval $T$ discretized in $N$ time nodes, resulting in the dynamic equation

$$
\boldsymbol{D}_{S} \boldsymbol{z}=\boldsymbol{u}
$$

where $2 n$ time boundary conditions are imposed by transferring the corresponding columns of the assembled matrix $\boldsymbol{D}_{\boldsymbol{S}}$ to the right-hand side of Eq. (6) after multiplying by the vector $\boldsymbol{U}_{c}$ of those conditions, resulting the equation

$$
\hat{\boldsymbol{K}}_{S} \hat{\boldsymbol{U}}=\hat{\boldsymbol{u}}, \quad \hat{\boldsymbol{u}}=\boldsymbol{u}-\boldsymbol{D}_{c} \boldsymbol{U}_{c}
$$

That can be solved to obtain the dynamic response, $\hat{U}$, of the human head model.

The time discretization used consisted of 40 temporal finite elements of $0.002 \mathrm{~s}$ in length, resulting in a total time of analysis of $0.08 \mathrm{~s}$.
The boundary conditions $\boldsymbol{U}_{c}$, of equation (7), were given as to model an initial translation with a velocity of $8.5 \mathrm{~m} / \mathrm{s}$, to be compatible with the study presented in reference [8], in which, the most common interval of head impact velocities in motorcycle helmets is $5,83-8,33 \mathrm{~ms}^{-1}$.

The discretized control force, appears in vector $\boldsymbol{u}$, of equation (6). Because the temporal finite elements consider the force in the beginning, middle and the end of each of the 40 time finite element, as can be seen in equation (4), we have 81 control variables to be calculated in the optimal control problem, as described in the next section.

\section{OPTIMISATION PROBLEM}

\section{A. Performance Criteria}

Several criteria were used to evaluate the performance of the isolation provided by the control force.

The maximum power developed in the area of the skull that is impacted can be quantified by Equation (8):

$$
J_{1}(u)=\max _{t}\left|k\left(x_{1}(t)-x_{3}(t)\right)\left(\dot{x}_{1}(t)-\dot{x}_{3}(t)\right)\right|
$$

The maximum acceleration reached by the brain, as given by Equation (9):

$$
J_{2}(u)=\max _{t}\left|\ddot{x}_{2}(t)\right|
$$

The maximum displacement of the skull, as given by Equation (10), is also used as a measure of the performance of the control force. This criteria can be interpreted as the thickness of the helmet liner.

$$
J_{3}(u)=\max _{t}\left|x_{1}(t)\right|
$$

Finally, the last criteria used is the Head Injury Criteria, which considers, not only the maximum acceleration attained by the head, but also the duration of the acceleration impulse. The expression that defines this criteria is given by Equation (11):

$$
H I C=\max _{t_{2}-t_{1}}\left\{\left[\frac{1}{t_{2}-t_{1}} \int_{t_{1}}^{t_{2}} \frac{\ddot{x}_{2}(t)}{g} d t\right]^{2,5}\left(t_{2}-t_{1}\right)\right\}
$$

Where the floating time interval $t_{2}-t_{1}$ is kept constant at $16 \mathrm{~ms}$ and is chosen as to maximize the quantity in brackets. In this work, HIC was calculated with non-overlapping time intervals. 


\section{B. Formulation of the Multicriteria Optimal Control Problem}

To deal with the various criteria of interest described in the previous section, one of them, the Head Injury Criteria, was chosen as the criteria to be minimized. The other three criteria were transformed in to inequality constraints and required to remain below a safety threshold value. So, the multicriteria control optimization problem can be formulated as follows:

$$
\min _{\mathrm{u}}\left\{H I C(u) \mid J_{1} \leq D_{1}, \quad J_{2} \leq D_{2}, \quad J_{3} \leq D_{3}\right\}
$$

With the threshold values of $\mathrm{J}_{1}, \mathrm{~J}_{2}$ and $\mathrm{J}_{3}$ chosen as $6210 \mathrm{~W}$, $200 \mathrm{~g}$ 's, and $18 \mathrm{~mm} \leq \mathrm{D}_{3} \leq 30 \mathrm{~mm}$

A limit value of $6210 \mathrm{~W}$ for criterion $\mathrm{J}_{1}$ indicates a $5 \%$ chance of occurrence of cranial fracture, The $200 \mathrm{~g}$ value for criterion $\mathrm{J}_{2}$ has been selected as a precaution, since according to the ECE 22.05 Helmet Safety Standard, the maximum permissible value for the resulting acceleration measured at the center of mass of the head in tests of safety helmets shall not at any time exceed $275 \mathrm{~g}$. The limit value for $\mathrm{J}_{3}$ was selected as values between $18 \mathrm{~mm}$ and $30 \mathrm{~mm}$, so as to provide optimal solutions for different lining thicknesses.

\section{OPTIMIZATION RESULTS}

The optimization problem of Equation (12) was solved using nonlinear programming. Three different values of the helmet liner thickness (D3) were selected: $18 \mathrm{~mm}, 20 \mathrm{~mm}$ and $30 \mathrm{~mm}$.

The optimal values of each performance criteria are summarized in table I. They give us the maximum value of the time evolution of each of the criteria that are represented in Figures 3 to 5 . As the admissible value of maximum displacement of the skull is relaxed from 18 to $30 \mathrm{~mm}$, one can see a decrease in each one of the performance criteria, which is as expected.

In every case, the maximum value of the HIC is well below the $2400 \mathrm{~g}^{2.5} \mathrm{~s}$ recommended by the ECE 22.05 Helmet Safety Standard.

In Figure 4, it can be seen that the higher the value of D3, the lowest the maximum acceleration of the brain, with a tendency to reduce the brain acceleration as time progresses, which is consistent with the time evolution of the control force, as shown in Figure 2.

By looking at Figure 5, one can see that, if D3 was to be relaxed even further, one would have to increase the total analysis time, because at $0.08 \mathrm{~s}$, the displacement of the skull would still be increasing. Even so, with D3 at $30 \mathrm{~mm}$, all the injury criteria already have safe values.

TABLE I. VAlues of Each Criteria at Each LeVel of D3

\begin{tabular}{|c|c|c|c|c|}
\hline $\mathbf{D 3}[\mathbf{m m}]$ & $\mathbf{J}_{\mathbf{1}}[\mathbf{W}]$ & $\mathbf{J}_{\mathbf{2}}[\mathbf{g}]$ & $\mathbf{J}_{\mathbf{3}}[\mathbf{m}]$ & $\left.\mathbf{H I C}_{[\mathbf{g}} \mathbf{g}^{\mathbf{2 . 5}} \mathbf{. s}\right]$ \\
\hline 18 & 6188.045 & 1961.992 & 0.018 & 810.0 \\
\hline 20 & 6210.011 & 1961.988 & 0.020 & 593.393 \\
\hline 30 & 5618.858 & 1644.043 & 0.030 & 245.026 \\
\hline
\end{tabular}

Figure 2. Optimal Control for Each Level of D3

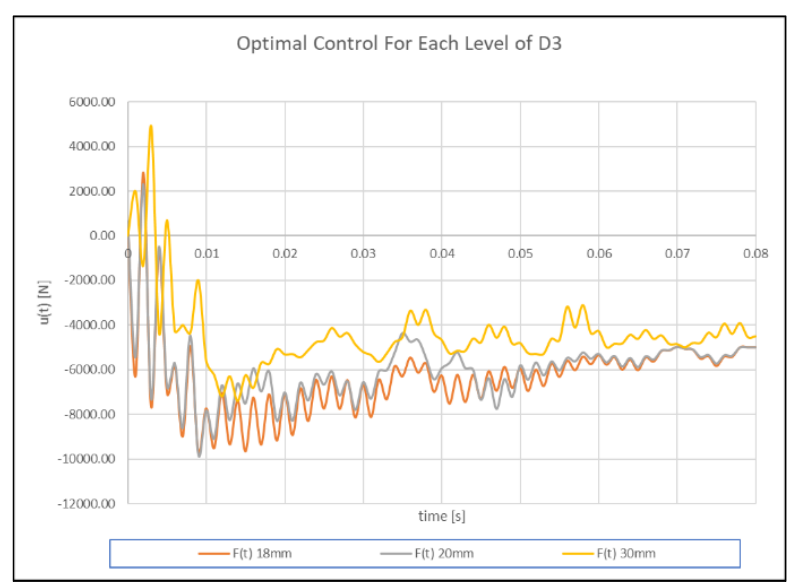

Figure 3. $J_{1}$ for Each Level of D3

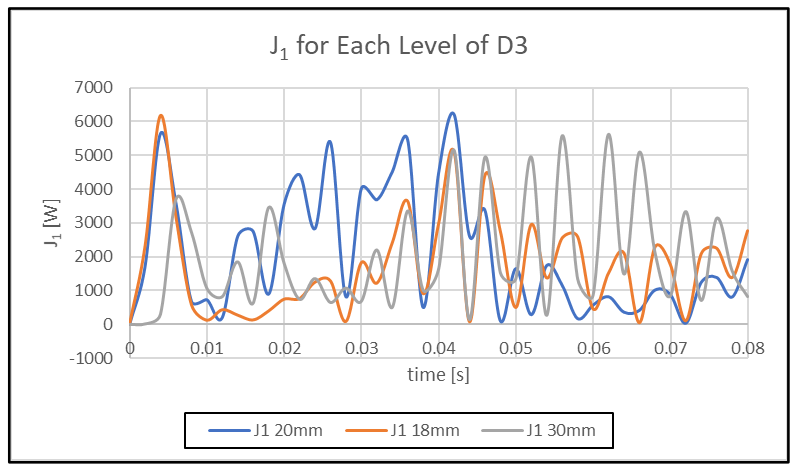

Figure 4. $J_{2}$ for Each Level of D3

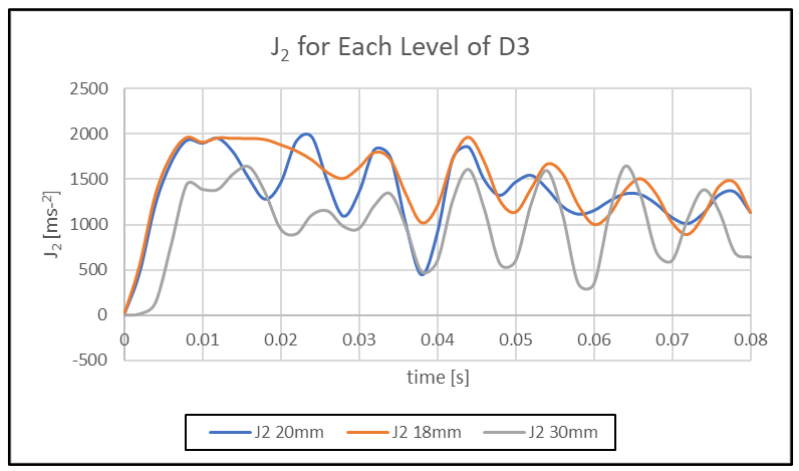

Figure 5. $J_{3}$ for Each Level of D3

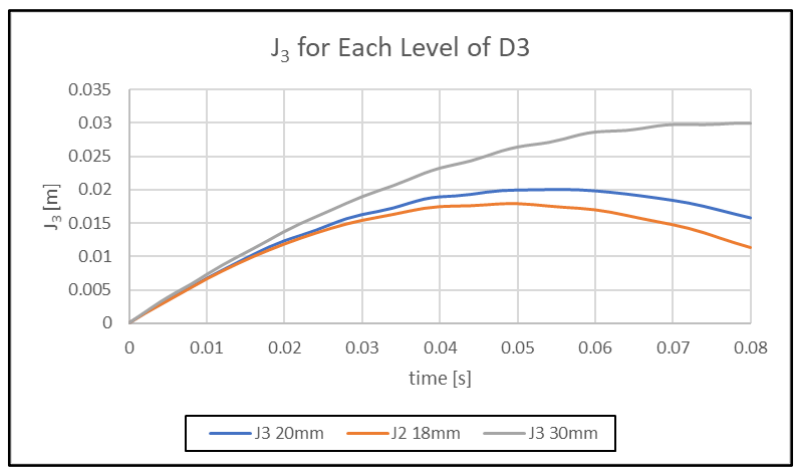




\section{CONCLUSION}

The limiting performance analysis of a head protection helmet was performed for various thicknesses of the helmet liner. The results obtained, although, not achievable by a real helmet, in the sense that the limiting performance analysis concept does not take into account any real-world engineering constraints in achieving the optimal control, are, even so, of interest. They provide a benchmark to which the performance of a real helmet can be compared to.

It is also revealed that, even with a very small $18 \mathrm{~mm}$ value of the helmet liner thickness, one can achieve injury criteria values bellow the safety threshold defined in section III.

\section{REFERENCES}

[1] C. MURRAY et al, "Global and regional mortality from 235 causes of death for 20 age groups in 1990 and 2010: a systematic analysis for the Global Burden of Disease Study 2010", The Lancet, vol. 380, pp. 2095-2128, 2012

[2] World Health Organization Report, Road Traffic Injuries Fact Sheet., http://www.who.int/en/news-room/fact-sheets/detail/road-trafficinjuries, 2018

[3] B. Chinn et al, "COST 327 - Motorcycle Safety Helmets. Final Report of the Action"., European Commission, Directory General for Energy and Transport, 2001.

[4] R. L. Stalnaker, A. C. Lin, and D. A. Guenther, "The Application of the New Mean Strain Criterion (NMSC)," Proceedings of the 1985 International IRCOBI/AAAM Conference on the Biomechanics of Impacts, Goteborg, Sweden, June 1985.

[5] M- Gellert, "A New Algorithm for Integration of Dynamic Systems", Computers \& Structures, 9, pp. 401-408, 1978.

[6] Z. Cheng, W. Pilkey, J. Crandall, C. Bass and K. Darvish, "Limiting Performance of Helmets for the Prevention of Head Injury", Shock and Vibration, 6(5-6), pp.299-320, 1999

[7] D. Balandin, N. Bolotnik, J. Crandall, W. Pilkey and S. Purtsezov, "Optimal Impact Isolation for Injury Prevention Evaluated by the Head Injury Criterion", Shock and Vibration, 14(5), pp.355-370, 2007

[8] M. Richter, D. Otte, U. Lehmann, B. Chinn, E. Schuller, D. Doyle, K. Sturrock and C. Krettek, "Head Injury Mechanisms in HelmetProtected Motorcyclists: Prospective Multicenter Study", The Journal of Trauma: Injury, Infection, and Critical Care, 51(5), pp.949-958, 2001.

[9] J. B. Cardoso, P. P. Moita and A. J. Valido, "Design and Control of Nonlinear Mechanical Systems for Minimum Time", Shock and Vibration, 15(3-4), pp. 315-324, 2008

[10] D.V. Balandin, N.N. Bolotnik and W.D. Pilkey, "Optimal Protection from Shock Impact and Vibration”, Gordon and Breach, 2001 